\title{
Non-lattice simulation of supersymmetric gauge theories as a probe to quantum black holes and strings
}

\author{
Jun Nishimura* \\ KEK Theory Center, Tsukuba 305-0801, Japan, \\ and Graduate University for Advanced Studies (SOKENDAI), Tsukuba 305-0801, Japan \\ E-mail: jnishi@post.kek.jp
}

In the past decade we have witnessed remarkable developments in the gauge-gravity duality, which suggested a new approach to superstring theory and quantum space-time. In this context it is important to study supersymmetric large- $N$ gauge theories in the strongly coupled regime. I will summarize the results and insights obtained so far by non-lattice simulations. A simple example of the gauge-gravity duality is the one between $1 \mathrm{~d} \mathrm{U}(N)$ gauge theory with 16 supercharges and the so-called black 0-brane solution in type IIA supergravity. In order for this duality to be valid, one has to take the 't Hooft large- $N$ limit and to take the strong coupling limit on the gauge theory side. The gauge theory can be regularized by fixing the gauge completely thanks to one dimension, and by introducing a Fourier mode cutoff. One can then use the standard RHMC algorithm to simulate the system. The energy calculated as a function of the temperature was compared with the results obtained from the gravity side based on the black hole thermodynamics. This confirmed the gauge-gravity duality with high accuracy and provided the microscopic origin of the black hole thermodynamics. From the calculation of the Wilson loop, one obtains the Schwarzschild radius of the dual geometry. One can actually use the present $1 \mathrm{~d}$ model with supersymmetric mass deformation to study $\mathscr{N}=4$ super Yang-Mills theory on $R \times S^{3}$ based on a novel large- $N$ reduction, which generalizes the original idea of Eguchi and Kawai. A test of this approach has been provided by Monte Carlo simulation at weak coupling. It is remarkable that we can now simulate the $4 \mathrm{~d}$ superconformal field theory, which appears in the most typical case of the gauge-gravity duality known as the AdS/CFT correspondence. In particular, no fine-tuning is required unlike previous proposals based on the lattice regularization.

The XXVII International Symposium on Lattice Field Theory - LAT2009

July 26-31 2009

Peking University, Beijing, China

\footnotetext{
* Speaker.
} 


\section{Introduction}

I hesitate a bit to talk about non-lattice simulation at this lattice conference, but this is indeed crucial for the purpose of simulating supersymmetric theories. What I am going to discuss is the so-called gauge-gravity duality, which is a conjecture from superstring theory [1], 2]. (See ref. [3] for a comprehensive review.) The statement itself is simple, and we don't even have to refer to superstring theory. Let us consider $\mathrm{U}(N)$ supersymmetric Yang-Mills theory (SYM) with 16 supercharges (or 32 supercharges, in a special case). We take the so-called 't Hooft limit, which amounts to sending $N$ to infinity with fixed $\lambda \equiv g_{\mathrm{YM}}^{2} N$. Next we consider the strongly coupled regime, namely the large- $\lambda$ regime. Then the statement is that the SYM is "dual" to a classical solution in 10d supergravity.

The argument for this conjecture is actually very intuitive and easy to understand. In superstring theories, there exists a soliton-like object termed D brane. "D" stands for the Dirichlet boundary condition imposed at the boundary of the worldsheet of a string. D brane can extend in $p+1$ dimensions, and it is characterized as a hypersurface on which strings can end on. Let us consider an open string attached to the $\mathrm{D}$ brane propagating along it. In figure 1 on the left, we describe such a process diagrammatically. If one slices the diagram in the orthogonal direction, one notices that the same process can be viewed as emission of a closed string. This is an example of the wellknown notion of open-string/closed-string duality. Note here that an open string includes a gauge particle as a massless mode, and similarly a closed string includes a graviton. Let us consider $N \mathrm{D}$ branes lying on top of each other. Then, in the low energy limit, one obtains $(p+1)$-dimensional $\mathrm{U}(N)$ SYM as an effective theory which describes the massless degrees of freedom of open strings attached to the $\mathrm{D}$ branes. On the other hand, one obtains a curved $10 \mathrm{~d}$ space-time in the bulk since the $\mathrm{D}$ brane sources gravitons. In order for the supergravity to be valid as a low energy and classical description of superstring theory in the bulk, one has to take the so-called 't Hooft large- $N$ limit with fixed $\lambda \equiv g_{\mathrm{YM}}^{2} N$, and then to take the large- $\lambda$ limit. This is so, since the string loop corrections are suppressed by $1 / N$, whereas the $\alpha^{\prime}$ corrections, which are due to strings having finite extent, are suppressed by some powers of $1 / \lambda$.

Why is this duality interesting? First of all, it is a realization of an old idea by 't Hooft 那, which states that the large- $N$ gauge theory is equivalent to some classical string theory, although in those days people may not have anticipated that the string theory actually lives in a curved space-time. It is interesting that the curved space-time emerges from a gauge theory in a flat space. This aspect of the duality is often referred to as the emergent space-time. In the gaugegravity duality, one typically obtains the anti-de Sitter space. If one considers the gauge theory at finite temperature, one obtains a black-hole-like geometry [5, 6]. As applications, one can study strongly coupled gauge theories, which are relevant to hadron and condensed matter physics, from a curved space-time. One can also use the duality in the opposite direction, and try to explain the microscopic origin of the black hole thermodynamics in terms of gauge theory. An ultimate goal of the gauge-gravity duality is to construct a non-perturbative and background independent formulation of superstring theory by using gauge-theory degrees of freedom.

Since the gauge-gravity duality is a strong-weak duality, it is important to study gauge theories in the strongly coupled regime. Monte Carlo simulation can be a powerful tool for such purposes. However, the problem is that the gauge theories we are interested in have supersymmetry, which 

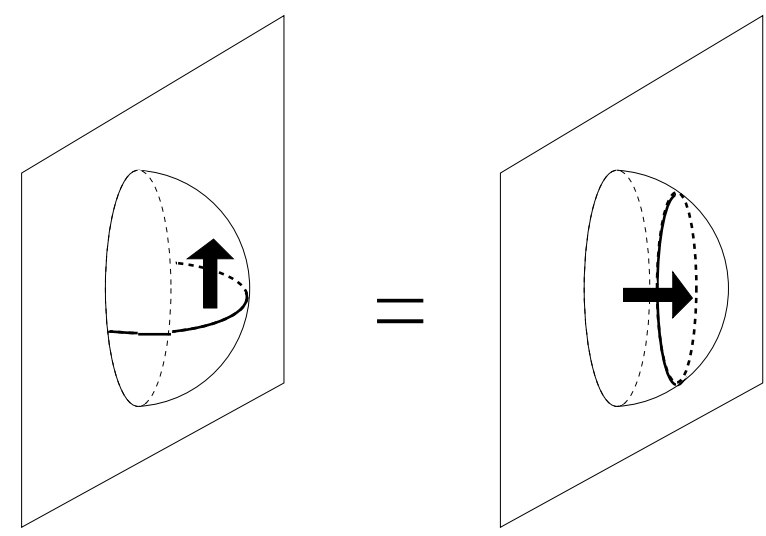

Figure 1: On the left, an open string attached to the D brane is propagating along it. On the right, the same process has been viewed as emission of a closed string from the D brane.

is broken by the lattice. This can be seen immediately if one recalls the supersymmetry algebra $\{Q, \bar{Q}\} \propto P_{\mu}$, where the generators for translation appear on the right hand side. Since the translational symmetry is broken by the lattice regularization, one necessarily breaks supersymmetry.

Recently there are considerable developments in "lattice supersymmetry", which can be categorized into two classes. One is the construction of lattice actions with various symmetries. For instance, one can preserve one supercharge by using the so-called topological twist. (See Catterall's contribution of this volume). The other one, which we discuss here, is non-lattice simulations 河 of supersymmetric gauge theories in 1 dimension with 16 supercharges [ [8, 9, 10]. Notably, one can extend this approach to $3 \mathrm{~d}$ and $4 \mathrm{~d}$ gauge theories [11] by using the idea of large- $N$ reduction [12]. In the $4 \mathrm{~d}$ case, the gauge theory becomes superconformal and the number of supersymmetries enhances from 16 to 32 . This superconformal theory is interesting on its own right, but it is also studied intensively in the context of the AdS/CFT correspondence, which is a typical case of the gauge-gravity duality [1]. The non-lattice simulation of the $4 \mathrm{~d}$ superconformal theory requires no fine-tuning, unlike the previous proposals based on the lattice regularization [13].

This article is organized as follows. In section 2 I discuss the non-lattice simulation of $1 \mathrm{~d}$ SYM with 16 supercharges. In particular, I explain how black hole thermodynamics appear from 1d SYM, and how the Schwarzschild radius appears from the Wilson loop. In section 3 I review the large- $N$ reduction, which enables us to extend these works to higher dimensions. In particular, I discuss how one can study $\mathscr{N}=4 \mathrm{SYM}$ on $R \times S^{3}$ in the 't Hooft limit, and present some preliminary results for the Wilson loop and the two-point correlation functions. In section $\emptyset$ I conclude with a summary.

\section{Non-lattice simulation of $1 d$ SYM with 16 supercharges}

The 1d SYM with 16 supercharges has the following actions for the bosonic part and the 
fermionic part, respectively.

$$
\begin{aligned}
& S_{\mathrm{b}}=\frac{1}{g^{2}} \int_{0}^{\beta} d t \operatorname{tr}\left\{\frac{1}{2}\left(D X_{i}(t)\right)^{2}-\frac{1}{4}\left[X_{i}(t), X_{j}(t)\right]^{2}\right\} \\
& S_{\mathrm{f}}=\frac{1}{g^{2}} \int_{0}^{\beta} d t \operatorname{tr}\left\{\frac{1}{2} \Psi_{\alpha} D \Psi_{\alpha}-\frac{1}{2} \Psi_{\alpha}\left(\gamma_{i}\right)_{\alpha \beta}\left[X_{i}, \Psi_{\beta}\right]\right\}
\end{aligned}
$$

It is a $1 \mathrm{~d} \mathrm{U}(N)$ gauge theory, and the covariant derivative is denoted as $D=\partial_{t}-i[A(t), \cdot]$. $X_{j}(t)(j=1, \cdots, 9)$ and $\Psi_{\alpha}(t)(\alpha=1, \cdots, 16)$ are $N \times N$ Hermitian matrices, and the theory has $\mathrm{SO}(9)$ symmetry. When we are interested in finite temperature, we impose periodic boundary conditions on $X_{j}(t)$ and anti-periodic boundary conditions on $\Psi_{\alpha}(t)$. Then the temperature is given by $T \equiv \beta^{-1}$, where $\beta$ is the extent in the Euclidean time $(t)$ direction. The 't Hooft coupling constant is defined by $\lambda \equiv g^{2} N$, which has the dimension of mass cubed. The physics of the system is determined only by the dimensionless coupling constant $\lambda_{\text {eff }} \equiv \frac{\lambda}{T^{3}}$. Therefore one can take $\lambda=1$ without loss of generality. With this convention, the low $T$ regime corresponds to the strongly coupled regime, which is expected to have the dual gravity description [ [5], whereas the high $T$ regime is essentially weakly coupled, and the high temperature expansion (HTE) is applicable [14].

In non-lattice simulation [7], we introduce an upper bound on the Fourier mode as $X_{i}(t)=$ $\sum_{n=-\Lambda}^{\Lambda} \tilde{X}_{i, n} \mathrm{e}^{i \omega n t}$, where $\omega=\frac{2 \pi}{\beta}$, and similarly for the fermions. This idea does not work usually because it breaks gauge invariance. (Recall that the Fourier mode is not a gauge invariant concept.) However, in 1d, one can fix the gauge non-perturbatively in the following way. We first take the static diagonal gauge $A(t)=\frac{1}{\beta} \operatorname{diag}\left(\alpha_{1}, \cdots, \alpha_{N}\right)$, in which the gauge field is constant in time and diagonal. By following the usual Faddeev-Popov procedure one obtains

$$
S_{\mathrm{FP}}=-\sum_{a<b} 2 \ln \left|\sin \frac{\alpha_{a}-\alpha_{b}}{2}\right|
$$

as a term to be added to the action. The above gauge choice does not fix the gauge symmetry completely, and there is a residual symmetry given by

$$
\alpha_{a} \mapsto \alpha_{a}+2 \pi v_{a}, \quad \tilde{X}_{i, n}^{a b} \mapsto \tilde{X}_{i, n-v_{a}+v_{b}}^{a b}, \quad \tilde{\Psi}_{\alpha, n}^{a b} \mapsto \tilde{\Psi}_{\alpha, n-v_{a}+v_{b}}^{a b},
$$

which represents a topologically nontrivial gauge transformation corresponding to the gauge function $g(t)=\operatorname{diag}\left(\mathrm{e}^{i \omega v_{1} t}, \cdots, \mathrm{e}^{i \omega v_{N} t}\right)$. This residual gauge symmetry can be fixed by imposing $-\pi<$ $\alpha_{a} \leq \pi$. One can then introduce the Fourier mode cutoff $\Lambda$. Since there is no UV divergence in this $1 \mathrm{~d}$ model, one can take the $\Lambda \rightarrow \infty$ limit naively, and one obtains the original gauge theory with 16 supercharges.

The system with finite $\Lambda$ can be simulated efficiently by using the standard RHMC algorithm [15]. In particular, the Fourier acceleration [16] can be implemented without extra cost since we are dealing with the Fourier modes directly as the fundamental degrees of freedom. This is crucial in reducing the critical slowing down at large $\Lambda$. (The same theory is also studied using the standard lattice approach [17]. However, from the results obtained so far, the non-lattice simulations seem to be far more efficient in obtaining the continuum limit.)

Let us first discuss the phase structure that appears when one changes the temperature. As is well known, the Polyakov line serves as an order parameter for the spontaneous breaking of 
the center symmetry. Figure 2 (Left) shows the results [8]. At high temperature the data agree nicely with the HTE [14] including the next-leading order. As the temperature decreases below $T \sim 3$, the data start to deviate, and at low temperature below $T \sim 0.9$, the data can be fitted to the characteristic behavior of the "deconfined phase"

$$
\langle|P|\rangle=\exp \left(-\frac{a}{T}+b\right) .
$$

In the temperature regime investigated, we find no phase transition. This is in sharp contrast to the bosonic model [18, 19, 20], which undergoes a phase transition to the "confined phase" at $T \sim 0.9$. The absence of the phase transition is consistent with analyses on the gravity side [5, 21].
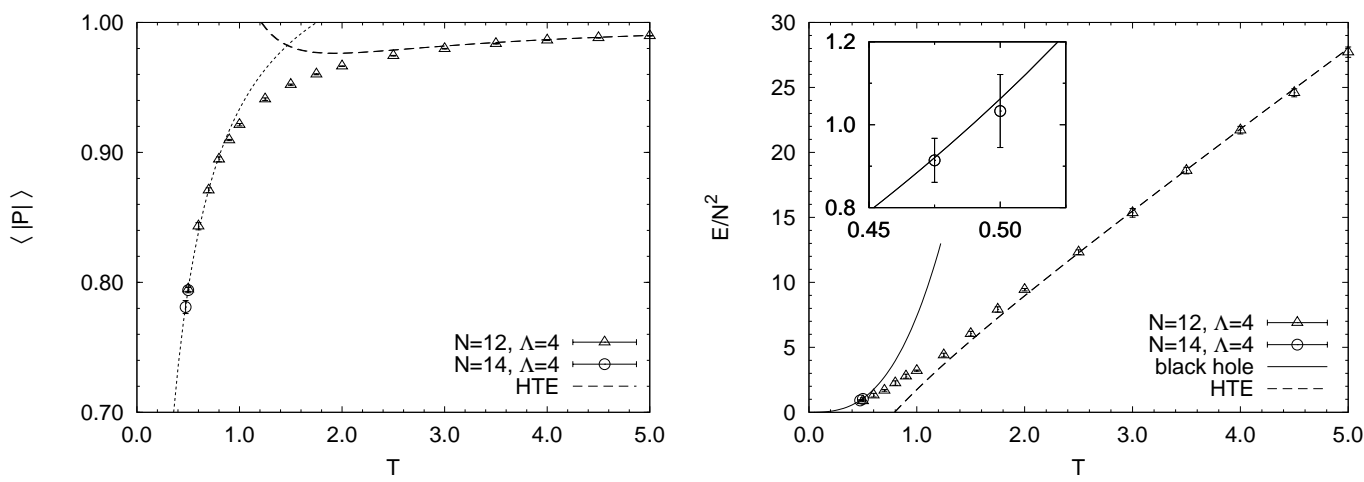

Figure 2: (Left) The Polyakov line is plotted against $T$. The dashed line represents the result of HTE up to the next leading order for $N=12$ [14]. The dotted line represents a fit to eq. (2.5) with $a=0.15$ and $b=0.072$. (Right) The energy (normalized by $N^{2}$ ) is plotted against $T$. The dashed line represents the result obtained by HTE up to the next leading order for $N=12$ [14]. The solid line represents the asymptotic power-law behavior at small $T$ predicted by the gauge-gravity duality. The upper left panel zooms up the region, where the power-law behavior sets in.

Let us turn to a quantitative prediction from the gauge-gravity duality. Given the dual geometry, one can use Hawking's theory of the black hole thermodynamics to obtain various thermodynamic relations such as [22]

$$
\frac{1}{N^{2}}\left(\frac{E}{\lambda^{1 / 3}}\right)=c\left(\frac{T}{\lambda^{1 / 3}}\right)^{14 / 5}, \quad c=\frac{9}{14}\left\{4^{13} 15^{2}\left(\frac{\pi}{7}\right)^{14}\right\}^{1 / 5}=7.41 \cdots
$$

The gauge-gravity duality predicts that this should be reproduced by 1d SYM in the large- $N$ limit at low $T$ [6]. The importance of this prediction is that, if it is true, it explains the microscopic origin of the black hole thermodynamics, meaning that the 1d SYM provides the quantum description of the states inside the black hole.

In figure 2 (Right) we plot the internal energy [8], which is defined by $E=\frac{\partial}{\partial \beta}(\beta \mathscr{F})$ in terms of the free energy $\mathscr{F}$. At $T \gtrsim 3$ the data agree with the HTE [14]. As one goes to lower temperature, the data points approach the solid line, which represents the result (2.6) obtained from the $10 \mathrm{~d}$ black hole. (See refs. [23] for earlier studies based on the Gaussian approximation.)

The plots in figure 1 were actually presented two years ago at LATTICE 2007 in Regensburg [24]. A common criticism in those days was that it was not clear whether the gauge theory results continue to follow the line predicted from gravity at lower $T$. In fact, simulations at lower $T$ 
are difficult, since one has to increase $\Lambda$ proportionally to $1 / T$, and at the same time one has to increase $N$ to avoid the run-away behavior due to finite $N$ [8]. Instead of lowering $T$, we were able to determine the power of the subleading term as [10]

$$
\frac{1}{N^{2}}\left(\frac{E}{\lambda^{1 / 3}}\right)=c\left(\frac{T}{\lambda^{1 / 3}}\right)^{14 / 5}-C\left(\frac{T}{\lambda^{1 / 3}}\right)^{23 / 5},
$$

from gravity. This was derived by considering higher derivative corrections in the supergravity action due to the effects of strings having finite extent ( $\alpha^{\prime}$ corrections). The coefficient $C$ of the subleading term is calculable in principle, but it requires the full information of the higher derivative corrections, which are yet to be determined. By using (2.7), however, we can already make a nontrivial test of the gauge-gravity duality [10]. In figure 3 (Left) we plot the discrepancy $7.41 T^{14 / 5}-E / N^{2}$ against $T$ in the $\log -\log$ scale, which reveals that the power of the subleading term is indeed consistent with the predicted value $23 / 5=4.6$. In figure 3 (Right) we find that the data at $T \lesssim 0.7$ can be nicely fitted to the form (2.7p with $C=5.58$. Note also that the $\Lambda=6$ data seem to suffer from some finite $\Lambda$ effects at low $T$. From this point of view, we consider that the $\Lambda=4$ data points at low $T$ in figure 2 (Right), which seem to be on the curve of the leading order result from gravity, also suffer from finite $\Lambda$ effects. Now we know that actually the subleading term in (2.7) should be taken into account for precise agreement.
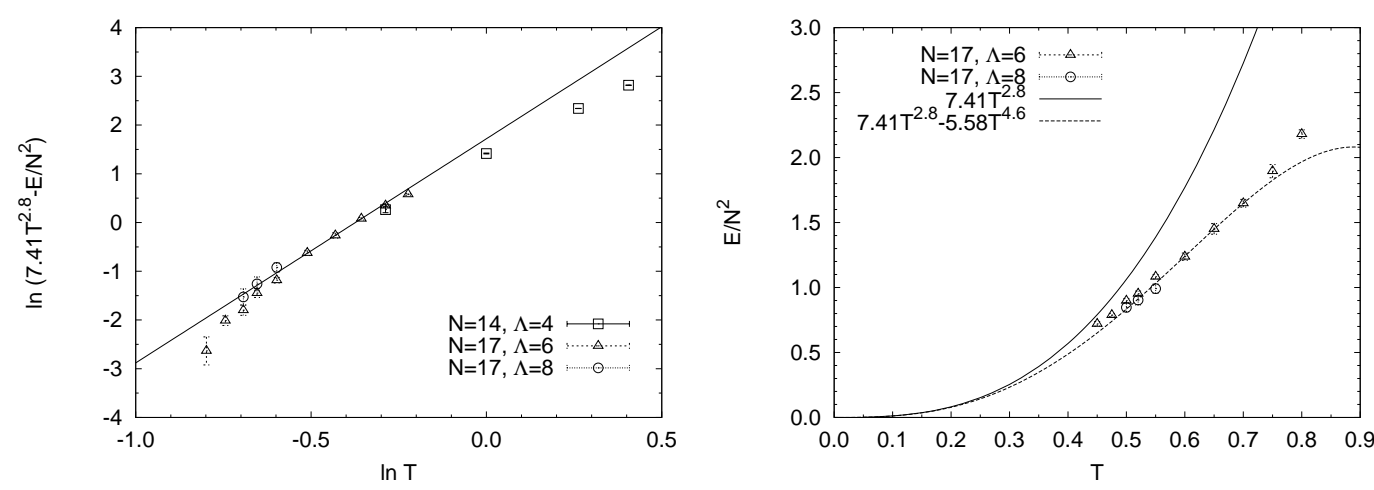

Figure 3: (Left) The deviation of the internal energy $\frac{1}{N^{2}} E$ from the leading term $7.41 T^{\frac{14}{5}}$ is plotted against the temperature in the $\log \log$ scale for $\lambda=1$. The solid line represents a fit to a straight line with the slope 4.6 predicted from the $\alpha^{\prime}$ corrections on the gravity side. (Right) The internal energy $\frac{1}{N^{2}} E$ is plotted against $T$ for $\lambda=1$. The solid line represents the leading asymptotic behavior at small $T$ predicted by the gauge-gravity duality. The dashed line represents a fit to the behavior (2.7) including the subleading term with $C=5.58$.

As another prediction from the gauge-gravity duality, let us consider the Wilson loop, which winds around the temporal direction once, like the Polyakov line. However, unlike the usual Polyakov line, we consider the one involving the adjoint scalar as

$$
W \equiv \frac{1}{N} \operatorname{tr} \mathscr{P} \exp \left[i \int_{0}^{\beta} d t\left\{A(t)+i n_{i} X_{i}(t)\right\}\right],
$$

where $n_{i}$ is a unit vector in $9 \mathrm{~d}$, which can be chosen arbitrarily due to the $\mathrm{SO}(9)$ invariance. This object can be calculated on the gravity side by considering the minimal surface spanning the loop 
in the dual geometry [25, 26]. For the present model, the result is given by [9]

$$
\ln W=\frac{\beta R_{\mathrm{Sch}}}{2 \pi \alpha^{\prime}}=\kappa\left(\frac{T}{\lambda^{1 / 3}}\right)^{-3 / 5},
$$

where $R_{\text {Sch }}$ is the Schwarzschild radius of the dual black hole geometry and

$$
\kappa=\frac{1}{2 \pi}\left\{\frac{16 \sqrt{15} \pi^{7 / 2}}{7}\right\}^{2 / 5}=1.89 \cdots .
$$

In figure 4 we plot the log of the Wilson loop [9] against $T^{-3 / 5}$ anticipating (2.9). Indeed, at low temperature (to the right on the figure), we find that the data points can be fitted nicely to a straight line with a slope 1.89 in precise agreement with (2.10). The solid line corresponds to $\langle\log |W|\rangle=1.89 T^{-3 / 5}-4.58$, where the existence of the constant term can be understood as $\alpha^{\prime}$ corrections. This result demonstrates that one can extract the information of the dual geometry such as the Schwarzschild radius from the gauge invariant observable (2.8). It also confirms directly [9] the fuzz-ball picture [27] of a black hole proposed to solve the information paradox.

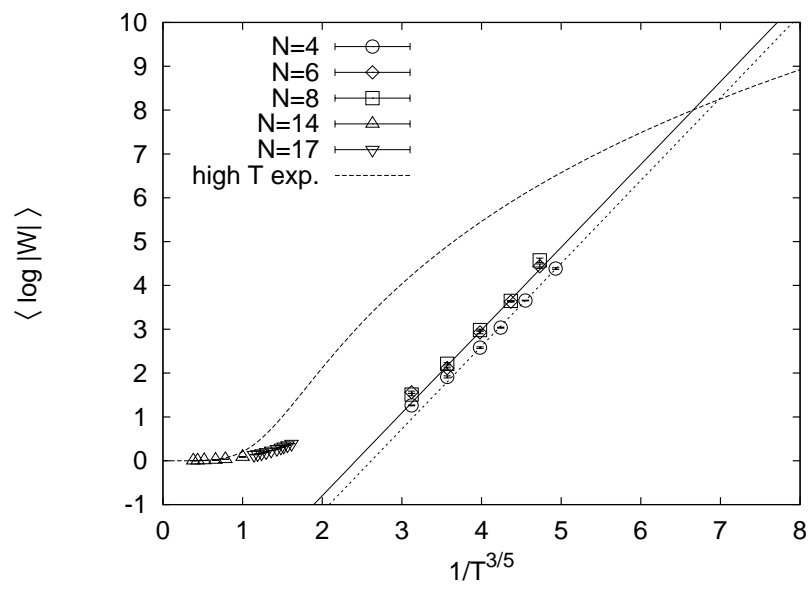

Figure 4: The plot of $\langle\log |W|\rangle$ for $\lambda=1$ against $T^{-3 / 5}$. The cutoff $\Lambda$ is chosen as follows: $\Lambda=12$ for $N=4 ; \Lambda=0.6 / T$ for $N=6,8 ; \Lambda=4$ for $N=14 ; \Lambda=6$ for $N=17$. The dashed line represents the results of the HTE up to the next-leading order for $N=14$, which are obtained by applying the method in Ref. [14]. The solid line and the dotted line represent fits for $N=6$ and $N=4$ respectively, to straight lines with the slope 1.89 predicted from the gravity side at the leading order.

One can also predict various correlation functions from gravity. This was done ten years ago by Sekino and Yoneya [28] extending the Gubser-Klebanov-Polykov-Witten prescription [2] to the present case. For instance, let us consider an operator

$$
\mathscr{O}_{\ell}=\operatorname{Str}\left(X_{i_{1}} X_{i_{2}} \cdots X_{i_{\ell}}\right),
$$

where the symbol " $\mathrm{S}$ " implies that all the indices are symmetrized. The two-point correlation function of this operator is predicted as

$$
\left\langle\mathscr{O}_{\ell}(t) \mathscr{O}_{\ell}(0)\right\rangle \sim \frac{1}{|t|^{p}}, \quad p=\frac{4 \ell-9}{5}
$$


at $\lambda^{-1 / 3} \ll|t| \ll \lambda^{-1 / 3} N^{10 / 21}$. In figure 5 we plot the two-point correlation function for $\ell=4,5$, which agrees precisely with the predicted power law behavior. See Ref. [29] for more details as well as results for other operators.

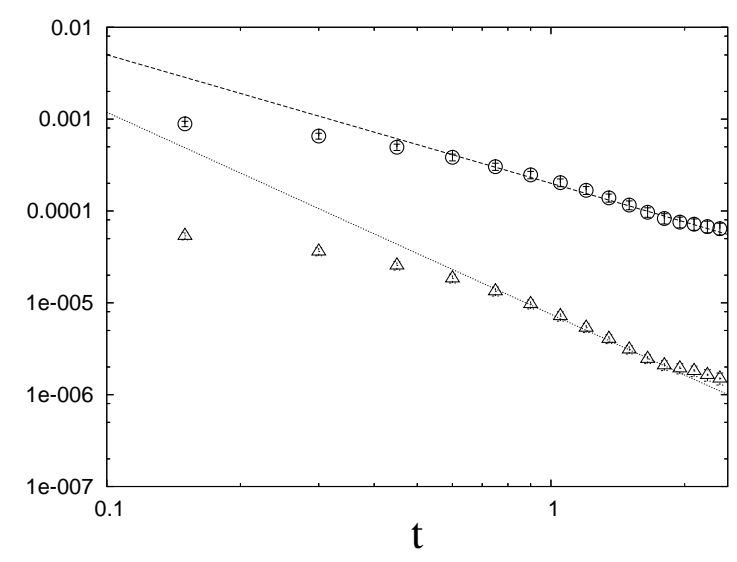

Figure 5: The two-point correlation function $\left\langle\mathscr{O}_{\ell}(t) \mathscr{O}_{\ell}(0)\right\rangle$ is plotted for $\ell=4$ (circles) and $\ell=5$ (triangles) in the $\log \log$ scale. Simulations were carried out at $N=3, \Lambda=12, T=0.2$. The straight lines are fits to the predicted power-law behavior (2.12).

\section{Extension to higher dimensions based on the large- $N$ reduction}

In this section we discuss how one can extend the works in the previous section to higher dimensions. Respecting supersymmetry becomes more non-trivial in higher dimensions, but here again we stick to a non-lattice regularization. For that purpose we use the idea of the large- $N$ reduction, which we review briefly. Let us consider $\mathrm{U}(N)$ gauge theory on a $D$-dimensional torus, and consider the Wilson loop defined by

$$
W[C]=\left\langle\mathscr{P} \exp \left(i \int A_{\mu}(x(\sigma)) \dot{x}^{\mu}(\sigma) d \sigma\right)\right\rangle,
$$

where the loop $C$ is specified by the embedding function $C=\left\{x^{\mu}(\sigma)\right\}$. The corresponding large- $N$ reduced model can be obtained by simply reducing the torus to a point. This implies that we drop the $x$-dependence of the field $A_{\mu}(x)$ and obtain $A_{\mu}$. The Wilson loop in the reduced model can be defined by

$$
w[C]=\left\langle\mathscr{P} \exp \left(i \int A_{\mu} \dot{x}^{\mu}(\sigma) d \sigma\right)\right\rangle_{\text {red }} .
$$

Then the statement is that

$$
\lim _{N \rightarrow \infty} w[C]=\lim _{N \rightarrow \infty} W[C] .
$$

The original idea was formulated on the lattice by Eguchi and Kawai [30]. However, it was soon pointed out by Bhanot, Heller and Neuberger [31] that there was a problem due to the spontaneous breaking of the center symmetry, which invalidates the proof of the statement. Several years ago Narayanan and Neuberger [32] proposed to avoid the spontaneous breaking of the center symmetry 
by not reducing the torus to a point completely, but keeping the volume finite in physical units. More recently, Kovtun, Ünsal and Yaffe pointed out [33] that the original proposal with the onesite model actually works by adding an adjoint fermion if its mass is sufficiently small, which was supported by numerical simulation [34]. See Bringoltz and Sharpe's contributions as well as Hietanen's one on this volume. (See also ref. [35] for a proposal for any non-abelian gauge theory.)

Here we use the idea of the large- $N$ reduction in order to study $\mathscr{N}=4 \mathrm{SYM}$ on $R \times S^{3}$ as proposed by Ref. [12]. It actually differs from the original large- $N$ reduction in that one deals with a curved space rather than a torus, which is a flat space. The theory obtained after reducing the $S^{3}$ to a point is given by the $1 \mathrm{~d}$ SYM, which is nothing but the one discussed in the previous section, plus some mass deformation, which preserves 16 supersymmetries of the undeformed theory. The additional terms are given by

$$
\int d t \operatorname{tr}\left[\frac{1}{2} \mu^{2} \sum_{i=1}^{3}\left(X_{i}\right)^{2}+\frac{1}{8} \mu^{2} \sum_{a=4}^{9}\left(X_{a}\right)^{2}+i \mu \varepsilon_{i j k} X_{i} X_{j} X_{k}+\frac{3}{8} i \mu \Psi \gamma_{123} \Psi\right],
$$

where $\mu$ is the deformation parameter, which is related to the radius of the $S^{3}$ before reduction as

$$
R_{\mathrm{S}^{3}}=\frac{2}{\mu} .
$$

This mass deformed theory possesses many classical vacua given by $X_{i}=\mu L_{i}$, where $L_{i}$ is an arbitrary (not necessarily irreducible) representation matrix of the $\mathrm{SU}(2)$ algebra $\left[L_{i}, L_{j}\right]=i \varepsilon_{i j k} L_{k}$. These vacua preserve 16 supersymmetries, and they are all degenerate.

In order to retrieve the original $4 \mathrm{~d} \mathscr{N}=4 \mathrm{SYM}$, one has to pick up a particular vacuum

$$
X_{i}=\mu\left(\begin{array}{llll}
L_{i}^{(n)} & & & \\
& L_{i}^{(n+1)} & & \\
& & \ddots & \\
& & & L_{i}^{(n+v-1)}
\end{array}\right) \otimes \mathbf{1}_{k}
$$

where $L_{i}^{(m)}$ represents the $m$-dimensional irreducible representation of the SU(2) algebra. Note, in particular, that there is an identity

$$
\sum_{i=1}^{3}\left(L_{i}^{(m)}\right)^{2}=\frac{1}{4}\left(m^{2}-1\right) \mathbf{1}_{m}
$$

which implies that each of $L_{i}^{(m)}$ in (3.6) represents a fuzzy sphere with the radius $\frac{\mu}{2} \sqrt{m^{2}-1}$. In this construction one regards the $S^{3}$ as an $S^{1}$ fiber on $S^{2}$, where the $S^{2}$ is represented by a fuzzy sphere, and the $S^{1}$ fibration is represented by having many of them with different radii. A more detailed argument for the reduction is given in ref. [12]. The statement is that in the $k \rightarrow \infty, n \rightarrow \infty$ and $v \rightarrow \infty$ limits, one obtains the $\mathscr{N}=4 \mathrm{U}(\infty) \mathrm{SYM}$ on $R \times S^{3}$, where the radius of $S^{3}$ is given by (3.5) and the 't Hooft coupling constant is given by

$$
\lambda_{\mathrm{SYM}}=2 \pi^{2}\left(R_{\mathrm{S}^{3}}\right)^{3} \frac{g^{2} k}{(2 n+v-1) / 2} .
$$


Note that one does not introduce the lattice structure anywhere in the formulation, which is important for preserving supersymmetry.

A check of this novel large- $N$ reduction has been provided [11] in the weak coupling limit by studying the deconfinement transition at finite temperature. Figure 5 shows that the results obtained from the reduced model with the background (3.6) reproduce the known result [36] for $\mathscr{N}=4 \mathrm{U}(\infty) \mathrm{SYM}$ on $R \times S^{3}$ in the $k, n, v \rightarrow \infty$ limit.

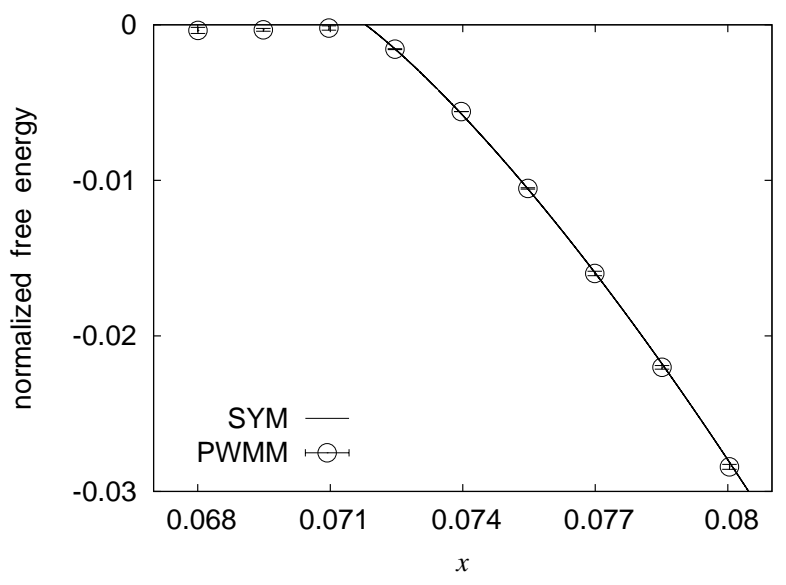

Figure 6: The normalized free energy of the reduced model around the background (3.6) with $n=\frac{v+1}{2}$ in the $k \rightarrow \infty$ and $v \rightarrow \infty$ limits is plotted against the dimensionless parameter $x=\exp (-\mu / 2 T)$ representing temperature near the critical point $x_{c}=0.072$. The error bars represent the fitting error associated with the extrapolation. The solid line represents the result [36] for the $\mathscr{N}=4 \mathrm{U}(\infty) \mathrm{SYM}$ on $R \times S^{3}$.

In order to test the approach at strong coupling, let us consider the circular Wilson loop in $\mathscr{N}=4 \mathrm{U}(\infty) \mathrm{SYM}$ on $R^{4}$. The expectation value of the Wilson loop is calculated exactly for arbitrary coupling constant, and the result is given as [37]

$$
\begin{aligned}
\left\langle W_{\text {circular }}\right\rangle & =\sqrt{\frac{2}{\lambda_{\mathrm{SYM}}}} I_{1}\left(\sqrt{2 \lambda_{\mathrm{SYM}}}\right) \\
& \simeq \frac{e^{\sqrt{2 \lambda_{\mathrm{SYM}}}}}{\left(\frac{\pi}{2}\right)^{1 / 2}\left(2 \lambda_{\mathrm{SYM}}\right)^{3 / 4}} \quad \text { at } \lambda_{\mathrm{SYM}} \gg 1
\end{aligned}
$$

in terms of the modified Bessel function of the first kind. At strong coupling it agrees with the result obtained from the dual geometry [25].

Since the $\mathscr{N}=4 \mathrm{SYM}$ is conformally invariant, the theory on $R^{4}$ is equivalent to the theory on $R \times S^{3}$ through conformal mapping. The circular Wilson loop on $R^{4}$ is mapped to a great circle on $S^{3}$ at a point on $R$. (The size of the circular Wilson loop corresponds to the position of the point on $R$, and the dilatation invariance on $R^{4}$ corresponds to the translational invariance on $R$.) This Wilson loop can be represented in the large- $N$ reduced model in a simple way as

$$
W_{\text {circular }}=\frac{1}{N} \operatorname{tr}\left[\exp \left(i \frac{4 \pi}{\mu}\left\{X_{3}(t)+i X_{4}(t)\right\}\right)\right],
$$

where $t$ can be any value due to translational symmetry, and hence one can take an average over it to increase statistics. 
In figure 7 we present our preliminary results for the circular Wilson loop [38]. (Here after, we present results obtained by imposing periodic boundary conditions on fermions, since we are interested in zero temperature.) We also plot the all order result (3.9. Although the matrix size is obviously too small, our Monte Carlo results look promising. Note, in particular, that we already start to observe a bent from the weak coupling behavior towards the strong coupling behavior.

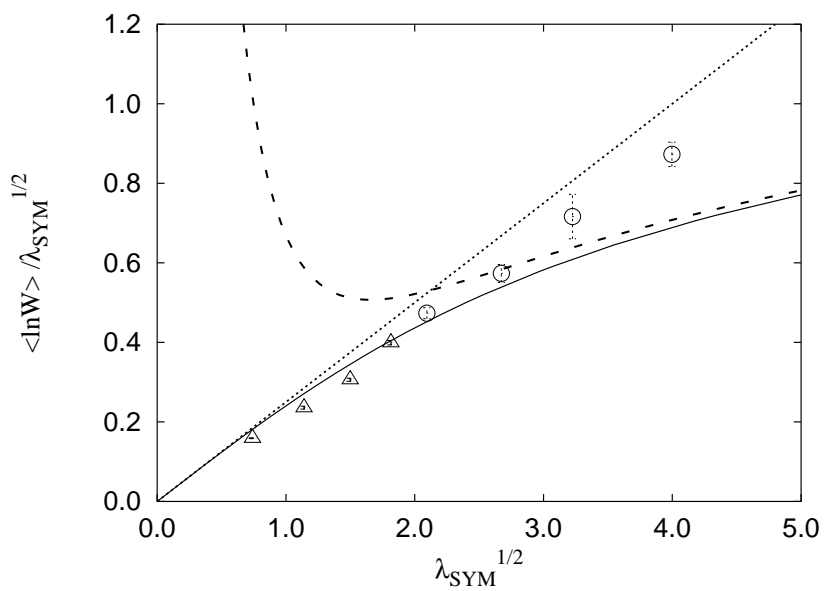

Figure 7: The log of the circular Wilson loop normalized by $\sqrt{\lambda_{\text {SYM }}}$ is plotted against $\sqrt{\lambda_{\mathrm{SYM}}}$. We have performed the $\Lambda \rightarrow \infty$ extrapolation (linear in $1 / \Lambda$ ) using $\Lambda=6,8,10$. The extent in the time direction is fixed to $\beta=5$. The background is chosen to be $n=1, v=2, k=2$ for $\sqrt{\lambda_{\mathrm{SYM}}}<2$, whereas for $\sqrt{\lambda_{\mathrm{SYM}}}>2$, we performed an extrapolation to $k=\infty$ using the data for $k=2,3$ assuming that the finite- $k$ effects are $\mathrm{O}\left(1 / k^{2}\right)$. The solid line represents the all order result (3.9). The dashed line represents the behavior 3.10) at strong coupling, whereas the dotted line represents the leading perturbative behavior $\ln \langle W\rangle \simeq \frac{1}{4} \lambda_{\text {SYM }}$.

Next let us consider chiral primary operators such as $\operatorname{tr} Z^{J}$, where $Z=\frac{1}{\sqrt{2}}\left(X_{4}+i X_{5}\right)$. The two-point function can be calculated in the weak coupling limit of $\mathscr{N}=4 \mathrm{U}(\infty) \mathrm{SYM}$ on $R^{4}$ as

$$
\left\langle\operatorname{tr} Z^{J}\left(z_{1}\right) \operatorname{tr} Z^{\dagger J}\left(z_{2}\right)\right\rangle_{R^{4}}=\frac{c_{J}}{\left|z_{1}-z_{2}\right|^{2 J}}, \quad c_{J}=J\left(\frac{\lambda_{\mathrm{SYM}}}{4 \pi^{2}}\right)^{J} .
$$

It is known in $\mathscr{N}=4 \mathrm{SYM}$ that the supersymmetry non-renormalization theorem holds for the two-point functions. Hence, the result (3.12) actually holds for arbitrary coupling constant. As in the case of the circular Wilson loop, one can make a conformal mapping to $R \times S^{3}$, and obtain for $J=2$, for instance,

$$
\int d \Omega_{3} \int d \Omega_{3}^{\prime}\left\langle\operatorname{tr} Z^{2}\left(t, \Omega_{3}\right) \operatorname{tr} Z^{\dagger 2}\left(0, \Omega_{3}^{\prime}\right)\right\rangle_{R \times S^{3}}=\frac{c_{2} e^{-\mu t}}{1-e^{-\mu t}},
$$

where we have integrated over the $S^{3}$ since the operator $\operatorname{tr} Z^{J}(t)$ in the reduced model corresponds to $\int d \Omega_{3} \operatorname{tr} Z^{J}\left(t, \Omega_{3}\right)$ in the $\mathrm{SYM}$ on $R \times S^{3}$.

In figure 8 we plot our preliminary results for $\left\langle\operatorname{tr} Z^{2}(t) \operatorname{tr} Z^{\dagger 2}(0)\right\rangle_{\text {red }}$ against the dimensionless time $\mu t$ [39]. Surprisingly our results for two different values of $\lambda_{\mathrm{SYM}}$ turn out to be very close to each other. This suggests that the non-renormalization theorem actually holds for each background. In fact we can obtain results in the weak coupling limit of the reduced model for the background 
(3.6), and show that the correlation function approaches the one (3.13) for the $\mathscr{N}=4 \mathrm{U}(\infty) \mathrm{SYM}$ in the $k, n, v \rightarrow \infty$ limit [39]. Therefore, if we are able to confirm the non-renormalization theorem for each background, it immediately implies that we can reproduce the results for the $\mathscr{N}=4 \mathrm{U}(\infty)$ SYM from the reduced model at arbitrary 't Hooft coupling constant.

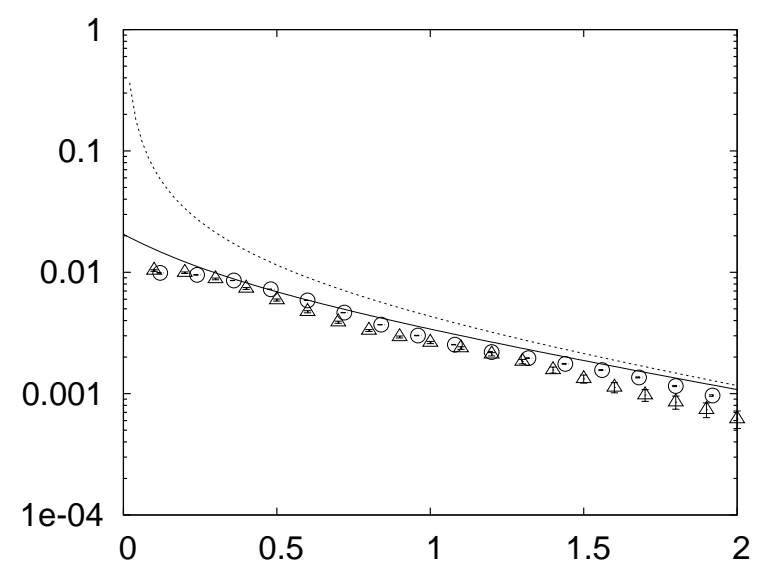

Figure 8: The two-point function of the chiral primary operator is calculated from the reduced model for a fixed background (3.6) with $n=3, v=2$ and $k=2$. The UV cutoff is chosen to be $\Lambda=10$. The circles represent results for $\mu=3, \beta=4$, which corresponds to $\lambda_{\mathrm{SYM}}=0.24$. The triangles represent results for $\mu=1, \beta=10$, which corresponds to $\lambda_{\mathrm{SYM}}=6.4$. Surprising, the results lie more or less on top of each other. The solid line represents the analytic result in the weak coupling limit of the reduced model for the same background in the $\Lambda \rightarrow \infty$ and $\beta \rightarrow \infty$ limits, which shows reasonable agreement with the Monte Carlo data. The dotted line represents the analytic result in the weak coupling limit of the $\mathscr{N}=4 \mathrm{U}(\infty) \mathrm{SYM}$, which is expected to be reproduced from the reduced model in the $k, n, v \rightarrow \infty$ limit.

What we have presented so far should be considered as a check of our method. More interesting quantities are those which are not obtained in the strongly coupled gauge theory, and yet there exist interesting predictions from gravity. Calculating such quantities by our method will clearly provide a new test of the AdS/CFT correspondence.

For instance, we can consider the rectangular Wilson loop in $R^{4}$, which behaves as

$$
\langle W(T \times R)\rangle=\exp \left(\frac{\gamma T}{R}\right)
$$

at $T \gg R$ due to conformal symmetry. This is in striking contrast to the area law in pure Yang-Mills theory. In particular, the AdS/CFT correspondence predicts

$$
\gamma=\frac{4 \pi^{2} \sqrt{2 \lambda_{\mathrm{SYM}}}}{\Gamma^{4}(1 / 4)}
$$

at strong coupling [25].

It would be also interesting to study higher point functions of the chiral primary operators. (See ref. [40] for calculation of extremal 3-point functions by simulating a "truncated theory" composed of six commuting bosonic matrices.) In particular, AdS/CFT predicts that non-extremal 4-point functions violate the non-renormalization theorem. It is interesting to check whether this is indeed the case, and to obtain explicit results, which can be compared with the prediction from gravity. 


\section{Summary}

I hope I have convinced the readers that non-lattice simulations are indeed useful for studying supersymmetric large- $N$ gauge theories in the strongly coupled regime. In the first part, I discussed the 1d SYM with 16 supercharges, which reproduced black hole thermodynamics and the Schwarzschild radius of the dual geometry. These results revealed direct connections to gravity as predicted by the gauge-gravity duality. In particular, the gauge theory results provided a clear understanding of the microscopic origin of the black hole thermodynamics. In the second part, I discussed how one can extend these works to higher dimensions by using the novel large- $N$ reduction. I have presented some preliminary results for $\mathscr{N}=4 \mathrm{U}(\infty) \mathrm{SYM}$ on $R \times S^{3}$. This theory is superconformal, and it actually has 32 supersymmetries. Our formulation preserves 16 supersymmetries in the $\Lambda \rightarrow \infty$ limit, and the remaining half of the supersymmetries are expected to be restored without fine-tuning by increasing the matrix size.

In a way, what we have seen is the beginning of a whole new field of research analogous to the situation of the lattice gauge theory in early 80s. Now with the aid of supersymmetry and large $N$, we have just started to explore superstring theory and quantum space-time from first principles.

\section{References}

[1] J. M. Maldacena, The large N limit of superconformal field theories and supergravity, Adv. Theor. Math. Phys. 2 (1998) 231 [hep-th/9711200].

[2] S. S. Gubser, I. R. Klebanov and A. M. Polyakov, Gauge theory correlators from non-critical string theory, Phys. Lett. B 428 (1998) 105 [hep-th/9802109].

E. Witten, Anti-de Sitter space and holography, Adv. Theor. Math. Phys. 2 (1998) 253 [hep-th/9802150].

[3] O. Aharony, S. S. Gubser, J. M. Maldacena, H. Ooguri and Y. Oz, Large N field theories, string theory and gravity, Phys. Rept. 323 (2000) 183 [hep-th/9905111].

[4] G. 't Hooft, A planar diagram theory for strong interactions, Nucl. Phys. B 72 (1974) 461.

[5] E. Witten, Anti-de Sitter space, thermal phase transition, and confinement in gauge theories, Adv. Theor. Math. Phys. 2 (1998) 505 [hep-th/9803131].

[6] N. Itzhaki, J. M. Maldacena, J. Sonnenschein and S. Yankielowicz, Supergravity and the large $N$ limit of theories with sixteen supercharges, Phys. Rev. D 58 (1998) 046004 [hep-th/9802042].

[7] M. Hanada, J. Nishimura and S. Takeuchi, Non-lattice simulation for supersymmetric gauge theories in one dimension, Phys. Rev. Lett. 99 (2007) 161602 [arXiv:0706.1647 [hep-lat ] ].

[8] K. N. Anagnostopoulos, M. Hanada, J. Nishimura and S. Takeuchi, Monte Carlo studies of supersymmetric matrix quantum mechanics with sixteen supercharges at finite temperature, Phys. Rev. Lett. 100 (2008) 021601 [arXiv: 0707.4454 [hep-th] ].

[9] M. Hanada, A. Miwa, J. Nishimura and S. Takeuchi, Schwarzschild radius from Monte Carlo calculation of the Wilson loop in supersymmetric matrix quantum mechanics, Phys. Rev. Lett. 102 (2009) 181602 [arXiv:0811.2081 [hep-th]].

[10] M. Hanada, Y. Hyakutake, J. Nishimura and S. Takeuchi, Higher derivative corrections to black hole thermodynamics from supersymmetric matrix quantum mechanics, Phys. Rev. Lett. 102 (2009) 191602 [arXiv:0811.3102 [hep-th]]. 
[11] G. Ishiki, S. W. Kim, J. Nishimura and A. Tsuchiya, Deconfinement phase transition in $\mathscr{N}=4$ super Yang-Mills theory on $R \times S^{3}$ from supersymmetric matrix quantum mechanics, Phys. Rev. Lett. 102 (2009) 111601 [arXiv:0810.2884 [hep-th] ];

Testing a novel large- $N$ reduction for $N=4$ super Yang-Mills theory on $R \times S^{3}, J H E P 09$ (2009) 029 [arXiv:0907.1488[hep-th]].

[12] T. Ishii, G. Ishiki, S. Shimasaki and A. Tsuchiya, $\mathscr{N}=4$ super Yang-Mills from the plane wave matrix model, Phys. Rev. D 78 (2008) 106001. [arXiv: 0807.2352 [hep-th]].

[13] D. B. Kaplan and M. Unsal, A Euclidean lattice construction of supersymmetric Yang-Mills theories with sixteen supercharges, JHEP 09 (2005) 042 [hep-lat / 0503039 ].

M. Unsal, Supersymmetric deformations of type IIB matrix model as matrix regularization of $\mathscr{N}=4$ SYM, JHEP 04 (2006) 002 [hep-th/ 0510004 ].

J. W. Elliott, J. Giedt and G. D. Moore, Lattice four-dimensional $\mathscr{N}=4$ SYM is practical, Phys. Rev. $D$ 78 (2008) 081701 [arXiv: 0806.0013 [hep-lat]].

[14] N. Kawahara, J. Nishimura and S. Takeuchi, High temperature expansion in supersymmetric matrix quantum mechanics, JHEP 12 (2007) 103 [arXiv: 0710.2188 [hep-th] ].

[15] M. A. Clark and A. D. Kennedy, The RHMC algorithm for 2 flavors of dynamical staggered fermions, Nucl. Phys. Proc. Suppl. 129 (2004) 850 [hep-lat/0309084].

[16] S. Catterall and S. Karamov, Testing a Fourier accelerated hybrid Monte Carlo algorithm, Phys. Lett. B 528 (2002) 301 [hep-lat/0112025].

[17] S. Catterall and T. Wiseman, Towards lattice simulation of the gauge theory duals to black holes and hot strings, JHEP 12 (2007) 104 [arXiv: 0706.3518 [hep-lat ] ];

Black hole thermodynamics from simulations of lattice Yang-Mills theory, Phys. Rev. D 78 (2008) 041502 [arXiv: 0803.4273 [hep-th] ];

Extracting black hole physics from the lattice, arXiv:0909.4947 [hep-th] .

[18] R. A. Janik and J. Wosiek, Towards the matrix model of M-theory on a lattice, Acta Phys. Polon. B 32 (2001) 2143 [hep-th/0003121];

P. Bialas and J. Wosiek, Towards the lattice study of M-theory. II, Nucl. Phys. Proc. Suppl. 106 (2002) 968 [hep-lat/0111034].

[19] O. Aharony, J. Marsano, S. Minwalla and T. Wiseman, Black hole - black string phase transitions in thermal 1+1 dimensional supersymmetric Yang-Mills theory on a circle, Class. Quant. Grav. 21 (2004) 5169 [hep-th/0406210].

[20] N. Kawahara, J. Nishimura and S. Takeuchi, Phase structure of matrix quantum mechanics at finite temperature, JHEP 10 (2007) 097 [arXiv: 0706.3517 [ hep-th ] ];

Exact fuzzy sphere thermodynamics in matrix quantum mechanics, JHEP 05 (2007) 091 [arXiv:0704.3183[hep-th]].

G. Mandal, M. Mahato and T. Morita, Phases of one dimensional large $N$ gauge theory in a 1/D expansion, arXiv:0910.4526[hep-th] .

[21] J. L. F. Barbon, I. I. Kogan and E. Rabinovici, On stringy thresholds in SYM/AdS thermodynamics, Nucl. Phys. B 544 (1999) 104 [hep-th/9809033].

O. Aharony, J. Marsano, S. Minwalla, K. Papadodimas, M. Van Raamsdonk and T. Wiseman, The phase structure of low dimensional large $N$ gauge theories on tori, JHEP 01 (2006) 140 [hep-th/0508077].

[22] I.R. Klebanov and A.A. Tseytlin, Entropy of near-extremal black p-branes, Nucl. Phys. B 475 (1996) $164[$ hep-th/9604089]. 
[23] D. Kabat, G. Lifschytz and D. A. Lowe, Black hole thermodynamics from calculations in strongly coupled gauge theory, Phys. Rev. Lett. 86 (2001) 1426 [hep-th/ 0007051 ]; Black hole entropy from non-perturbative gauge theory, Phys. Rev. D 64 (2001) 124015 [hep-th/ 0105171$].$

[24] J. Nishimura, K. N. Anagnostopoulos, M. Hanada and S. Takeuchi, Putting M theory on a computer, PoS LAT2007 (2007) 059 [arXiv: 0801 . 4205 [hep-lat] ].

[25] S. J. Rey and J. T. Yee, Macroscopic strings as heavy quarks in large $N$ gauge theory and anti-de Sitter supergravity, Eur. Phys. J. C 22 (2001) 379 [hep-th/9803001].

J. M. Maldacena, Wilson loops in large N field theories, Phys. Rev. Lett. 80 (1998) 4859 [hep-th/9803002].

[26] S. J. Rey, S. Theisen and J. T. Yee, Wilson-Polyakov loop at finite temperature in large N gauge theory and anti-de Sitter supergravity, Nucl. Phys. B 527 (1998) 171 [hep-th/9803135].

[27] S. D. Mathur, Fuzzballs and the information paradox: a summary and conjectures, arXiv:0810.4525[hep-th].

[28] Y. Sekino and T. Yoneya, Generalized AdS-CFT correspondence for matrix theory in the large $N$ limit, Nucl. Phys. B 570 (2000) 174 [hep-th/9907029].

[29] M. Hanada, J. Nishimura, Y. Sekino and T. Yoneya, Monte Carlo studies of Matrix theory correlation functions, arXiv:0911.1623 [hep-th].

[30] T. Eguchi and H. Kawai, Reduction of dynamical degrees of freedom in the large $N$ gauge theory, Phys. Rev. Lett. 48 (1982) 1063.

[31] G. Bhanot, U. M. Heller and H. Neuberger, The quenched Eguchi-Kawai model, Phys. Lett. B 113 (1982) 47.

[32] R. Narayanan and H. Neuberger, Large N reduction in continuum, Phys. Rev. Lett. 91 (2003) 081601 [hep-lat/0303023].

[33] P. Kovtun, M. Unsal and L. G. Yaffe, Volume independence in large N(c) QCD-like gauge theories," JHEP 06 (2007) 019 [hep-th/ 0702021$].$

[34] B. Bringoltz and S. R. Sharpe, Non-perturbative volume-reduction of large-N QCD with adjoint fermions, arXiv:0906.3538 [hep-lat].

[35] M. Unsal and L. G. Yaffe, Center-stabilized Yang-Mills theory: confinement and large $N$ volume independence, Phys. Rev. D 78 (2008) 065035 [arXiv: 0803.0344 ].

[36] O. Aharony, J. Marsano, S. Minwalla, K. Papadodimas and M. Van Raamsdonk, The Hagedorn / deconfinement phase transition in weakly coupled large $N$ gauge theories, Adv. Theor. Math. Phys. 8 (2004) 603 [hep-th/0310285].

[37] J. K. Erickson, G. W. Semenoff and K. Zarembo, Wilson loops in N = 4 supersymmetric Yang-Mills theory, Nucl. Phys. B 582 (2000) 155 [hep-th/ 0003055 ];

N. Drukker and D. J. Gross, An exact prediction of $N=4$ SUSYM theory for string theory, J. Math. Phys. 42 (2001) 2896 [hep-th/ 0010274 ];

V. Pestun, Localization of gauge theory on a four-sphere and supersymmetric Wilson loops, arXiv:0712.2824[hep-th].

[38] G. Ishiki, M. Honda, J. Nishimura, A. Tsuchiya, in progress.

[39] G. Ishiki, M. Honda, S. W. Kim, J. Nishimura, A. Tsuchiya, in progress.

[40] D. Berenstein, R. Cotta and R. Leonardi, Numerical tests of AdS/CFT at strong coupling, Phys. Rev. D 78 (2008) 025008 [arXiv: 0801.2739 [hep-th] ]. 